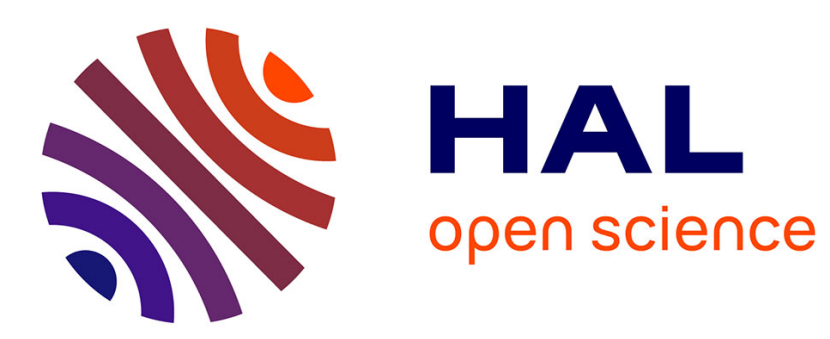

\title{
Spherical Polar Fourier EAP and ODF Reconstruction via Compressed Sensing in Diffusion MRI
}

\author{
Sylvain Merlet, Jian Cheng, Aurobrata Ghosh, Rachid Deriche
}

\section{To cite this version:}

Sylvain Merlet, Jian Cheng, Aurobrata Ghosh, Rachid Deriche. Spherical Polar Fourier EAP and ODF Reconstruction via Compressed Sensing in Diffusion MRI. ISBI, Mar 2011, Chicago, United States. inria-00585694

\section{HAL Id: inria-00585694 https://hal.inria.fr/inria-00585694}

Submitted on 13 Apr 2011

HAL is a multi-disciplinary open access archive for the deposit and dissemination of scientific research documents, whether they are published or not. The documents may come from teaching and research institutions in France or abroad, or from public or private research centers.
L'archive ouverte pluridisciplinaire HAL, est destinée au dépôt et à la diffusion de documents scientifiques de niveau recherche, publiés ou non, émanant des établissements d'enseignement et de recherche français ou étrangers, des laboratoires publics ou privés. 


\title{
SPHERICAL POLAR FOURIER EAP AND ODF RECONSTRUCTION VIA COMPRESSED SENSING IN DIFFUSION MRI
}

\author{
Sylvain Merlet ${ }^{1}$, Jian Cheng ${ }^{1,2}$, Aurobrata Ghosh ${ }^{1}$ and Rachid Deriche ${ }^{1}$ \\ ${ }^{1}$ Athena Project-Team, INRIA Sophia Antipolis - Méditerranée, France \\ ${ }^{2}$ Center for Computational Medecine, LIAMA, Institute of Automation, Chinese \\ Academy of Sciences, China
}

\begin{abstract}
In diffusion magnetic resonance imaging (dMRI), the Ensemble Average Propagator (EAP), also known as the propagator, describes completely the water molecule diffusion in the brain white matter without any prior knowledge about the tissue shape. In this paper, we describe a new and efficient method to accurately reconstruct the EAP in terms of the Spherical Polar Fourier (SPF) basis from very few diffusion weighted magnetic resonance images (DW-MRI). This approach nicely exploits the duality between SPF and a closely related basis in which one can respectively represent the EAP and the diffusion signal using the same coefficients, and efficiently combines it to the recent acquisition and reconstruction technique called Compressed Sensing (CS). Our work provides an efficient analytical solution to estimate, from few measurements, the diffusion propagator at any radius. We also provide a new analytical solution to extract an important feature characterising the tissue microstructure: the Orientation Distribution Function (ODF). We illustrate and prove the effectiveness of our method in reconstructing the propagator and the ODF on both noisy multiple q-shell synthetic and phantom data.
\end{abstract}

Index Terms - Diffusion MRI, Compressed sensing, Ensemble Average Propagator recovery, Propagator, Orientation Distribution Function, Spherical Polar Fourier.

\section{INTRODUCTION}

Diffusion MRI (dMRI) is a recent Magnetic Resonance Imaging technique introduced by $[1,2,3]$. It enables the quantification of water diffusion influenced by biological tissues. Hence, dMRI has become an established research tool in the investigation of tissue structure and orientation.

In 1965, [4] introduced the pulsed gradient spin-echo (PGSE) sequence. It allows the quantification of the diffusion by estimating the displacement of particles from the phase change that occurs during the acquisition process. When the gradient pulses are sufficiently short, [4] also showed that the measured signal $E(\mathbf{q})$, after normalization, is written as the Fourier transform of the EAP $P(\mathbf{R})$

$$
E(\mathbf{q})=\int P(\mathbf{R}) \exp (-2 \pi i \mathbf{q} \cdot \mathbf{R}) d \mathbf{R},
$$

where $\mathbf{q}$ and $\mathbf{R}$ are both $3 \mathrm{D}$-vectors that respectively represent the effective gradient direction and the displacement direction.

Many models, that require several DW-MRI, enable the EAP estimation. Diffusion Tensor Imaging (DTI) model requires at least 6 DW-MRIs plus an additional unweighted image. However, the Gaussian assumption, in DTI, is an oversimplification of the diffusion of water molecules in the brain and thus has some limitations. Other models, the High Angular Resolution Diffusion Imaging (HARDI) models, have been proposed to infer the diffusion of water molecules along several directions. One of the HARDI techniques known as the Q-ball imaging (QBI) [5, 6] consists of sampling the qspace on a single sphere in several gradient directions in order to estimate the ODF. The ODF $[5,6,7,8]$ is a spherical function that contains the angular information of the diffusion process. [6] found an elegant analytical solution by modelling the ODF using Spherical Harmonics (SH). More recently, [8] used the spherical ridgelets to approximate the ODF. An other model, the diffusion spectrum imaging (DSI), was developped to estimate the EAP by taking samples in the whole q-space. It aims at completely describing the EAP without any prior knowledge about the tissue shape. However, it is computationally expensive if we want to accurately reconstruct the EAP. Some "cheaper" techniques were developed to perform a complete (radial and angular part) estimation of the EAP from measurements taken on one or several shells. For instance, DOT [9], estimate the EAP using samples acquired on one shell. However, [9] assumes a monoexponential decay of the diffusion signal, which is not verified all the time. Reference [9] proposes an extent to a multiexponential decay model but is impractical because of the large number of samples required to estimate the radial part. Reference [10] suggests, with DPI, to use the general solution of the Laplace's equation to model both the radial and angular part of the EAP. In $[11,12]$, the authors approximate the attenuation signal in the SPF basis and extract different 
EAP features from this representation.

These techniques still need many measurements so we give attention on Compressed Sensing (CS), a recent technique to accurately reconstruct signals from under sampled measurements. CS, multiple shells HARDI and EAP play a central role in this work which is focused on the development of a CS based method for accelerating the acquisition by significantly reducing the number of measurements. CS has already been exploited in dMRI. For instance, [13] reconstruct the EAP in a numerical fashion with much less measurements and better results than DSI. [14] also use CS to model the ODF in terms of spherical ridgelet from highly reduced HARDI samples.

In this paper we describe a CS based EAP reconstruction from multiple shell acquisitions using the reciprocal relation between the SPF basis in real space and its dual basis in qspace. Our method significantly undersamples the q-space in a random fashion and then reconstructs the EAP by solving a convex optimization problem. The first section aims at presenting the background theory related to this work, i.e. the CS theory and the mathematical framework for the SPF basis. Then we provide the solution to estimate the EAP in SPF basis at any radius $R$. We also propose to analytically extract the ODF feature. The last section presents results on both noisy synthetic data and phantom data.

\section{BACKGROUND THEORY}

\subsection{Compressed Sensing}

The Compressed Sensing acquisition process has been proving useful in recovering Magnetic resonance (MR) images by significantly undersampling their k-spaces [15] [16] [17] [18]. David Donoho described a complete mathematical framework [19] and published a patent [20] of CS theory.

Suppose our signal of interest is a vector $\mathbf{x} \in \mathcal{R}^{m}$ and we are able to acquire an observation representative of $\mathbf{x}$. Usually, the way the measurement is done is constrained by the acquisition process specific for an application. In MR imaging, for instance, the samples are taken from the k-space, which represents the Fourier transform of a given MR image. In most applications, we need to decrease time in capturing data and, besides technological advance, the best way to achieve our goal is to decrease as most as possible the number of acquisitions. Let $\mathbf{y} \in \mathcal{R}^{n}$, with $n<<m$, be the measurement vector given by the sensor of a given application. $y$ obeys this equation:

$$
\mathbf{y}=\mathbf{A x}+\mathbf{z}
$$

Where $\mathbf{A} \in \mathcal{R}^{n \times m}$ is the measurement matrix, so called the CS matrix, and $\mathbf{z} \in \mathcal{R}^{n}$ represents the acquisition noise. Our goal is to find $\mathbf{x}$ given a measure $\mathbf{y}$. Since $\mathbf{y}$ has less entries than $\mathbf{x}$, this ill-posed problem cannot be resolved without any prior knowledge about the signal to recover.
This technique is, thus, based on the data sparsity or compressibility, i.e. that all the signal information can be contained in few signal coefficients. Conceptually, the CS method follows this idea : if the underlying signal is entirely described by a small number of coefficients then it is not necessary to acquire every data sample. Hence, a fundamental condition in the Compressed Sensing theory is that the signal admits a sparse representation. Many transforms are known to make a signal sparse. For instance we can cite the Discrete Wavelet Transform (DWT) or the Discrete Cosine Transform (DCT) respectively used in JPEG2000 and JPEG standard to compress natural images. A simple gradient transform is also known to compress piecewise constant signals. Suppose, we know an orthonormal basis $B$ of $\mathcal{R}^{m}$ adequate to represents $\mathrm{x}$. Then $\mathrm{x}$ can be modelled as a linear combination of the basis function $b \in B$, i.e. $\mathbf{x}=\sum_{b \in B}<x, b>b$. The transform coefficients $c=\langle x, b\rangle$ form a vector $\mathbf{c} \in \mathcal{R}^{n_{c}}$ describing $\mathbf{x}$ with respect to the basis $\mathbf{B}$. $\mathbf{c}$ is said sparse, or compressible, if the most important coefficients in a chosen sparse representation are much more smaller, in number, than $n_{c}$.

Another important point for CS is the degree of incoherent aliasing that appears after applying a sparse transform. [15] pointed out that the data acquisition scheme has to result in incoherent artefacts in the chosen sparse domain. A, usually called the CS matrix, reflects the way the measures are taken. In [15], since the measurements are a sub-set of the k-space samples, the CS matrix is an undersampled Fourier Operator. The CS matrix can be represented as follows :

$$
A=U D
$$

where $D$ is the matrix representation of a digital domain, as the complete Fourier operator when the measurements are kspace samples. $D$ can take many forms but, usually, they are restrained by the measurement sensor.

The matrix $U$ represents the way the acquisition is done. If the samples are taken in a random fashion, $U$ can have its entries generated by random numbers following a specific distribution. [20] tells about random projectors, i.e. matrices whose rows are orthogonalized by a standard procedure such that the Gram-Schmidt procedure or partial Hadamard matrices. In most cases, $U$ is part of a known transform and is not defined explicitly.

The CS reconstruction is based on a $l_{1}$ minimization scheme promoting the signal sparsity. Considering the signal admits a sparse representation with respect to an orthonormal basis $B$, we can constrain most of the transform coefficients $\left\{c_{i}=<x, b_{i}>, b_{i} \in B, i=1, \ldots, n_{c}\right\}$ to be zero by minimizing the number of non-zero elements, that is the $l_{0}$ norm defined by $\|\mathbf{c}\|_{0}=\sum_{i=1}^{n_{c}} c_{i}^{0}$. Nevertheless, minimizing the $l_{0}$ norm is a NP-complete problem and requires combinatorial optimization to approximate it, so we minimize the $l_{1}$ norm defined by $\|\mathbf{c}\|_{1}=\sum_{i=1}^{n_{c}}\left|c_{i}\right|[15] . l_{1}$ norm is just the 
sum of the absolute values of every element. The solution $\mathbf{x}$ of our problem is given by solving the following convex optimization problem :

$$
\operatorname{argmin}_{\mathbf{x}} J(\mathbf{x})=\|\mathbf{A x}-\mathbf{y}\|_{2}^{2}+\lambda\|\mathbf{c}\|_{1} .
$$

The first term is the data consistency constraint, $\|\mathbf{c}\|_{1}$ is the sparsity constraint. $\lambda$ is the Lagrange parameter that defines the confidence we put in the measured signal $y$. The data consistency constraint enables the solution to remain close to the raw data acquisition, whereas the minimization of the second term promotes sparsity. In short, this mathematical problem searches for the sparsest solution while remaining close to the acquired data.

\subsection{The Spherical Polar Fourier basis}

For three-dimensional signal described in spherical coordinate $(r, \theta, \varphi)$, it is useful to find a decomposition into functions that have appropriate radial and angular structure. Such a basis can be separated in two parts as

$$
\Psi(r, \theta, \varphi)=R(r) \Omega(\theta, \varphi)
$$

where $R$ and $\Omega$ respectively stand for the radial and angular part of the basis

We choose to represent the angular structure in term of the $\mathrm{SH}$ basis. These functions have been of great interest in estimating spherical functions [21, 6]. In QBI [6], only $15 \mathrm{SH}$ are usually sufficient to correctly estimate the fibres orientation. Thus, the SH basis is a good candidate to sparsely represent the angular part of our signal. The complex spherical harmonics are the solution of the angular part of the Laplace equation in spherical coordinates and are expressed as

$$
C_{\ell}^{m}(\theta, \varphi)=\sqrt{\frac{(2 \ell+1)}{4 \pi} \frac{(\ell-m) !}{(\ell+m) !}} P_{\ell}^{m}(\cos \theta) e^{i m \varphi},
$$

where $\ell \in \mathcal{N}$ is the spherical harmonic order and $-l \leq m \leq l$ the degree. Eq. 6 shows a complex function. However the EAP is assumed to be real and antipodally symmetric, so we use a modified version of this basis [6]. For $l=0,2,4, \ldots, L$ and $m=-l, \ldots, 0, \ldots, l$

$$
Y_{\ell}^{m}=\left\{\begin{array}{ll}
C_{\ell}^{0} & \text { if } m=0 \\
\frac{1}{\sqrt{2}}\left(C_{\ell}^{m}+(-1)^{m} C_{\ell}^{-m}\right) & \text { if } m>0 \\
\frac{1}{i \sqrt{2}}\left(C_{\ell}^{-m}-(-1)^{m} C_{\ell}^{m}\right) & \text { if } m<0 .
\end{array} .\right.
$$

The family of functions $Y_{\ell}^{m}$ form a real and orthonormal basis. The number of spherical harmonics for a given order $L$ is $J=(1 / 2)(L+1)(L+2)$.

Another transform, the spherical ridgelet transform [8, 14], appears promising in sparsely representing angular structures. About, radial decomposition of the three dimensional EAP, we use an orthonormal basis in spherical coordinates known as the Gauss-Laguerre (GL) functions. The GL basis function of order $n$ is expressed as

$$
R_{n}(r)=\left[\frac{2 n !}{\zeta^{3 / 2} \Gamma(n+3 / 2)}\right]^{1 / 2} \exp \left(\frac{-r^{2}}{2 \zeta}\right) L_{n}^{1 / 2}\left(\frac{r^{2}}{\zeta}\right)
$$

where $\zeta$ is a scale factor and $L_{n}^{1 / 2}$ is the generalized Laguerre polynomial of order $n$. [11] report that the GL basis is appropriate to sparsely represent the radial part of the Gaussianlike patterns. It can describe multiple configurations of the radial diffusion including isotropy and crossing fibres aspects as well as the multiple compartments profile.

The complete approximation of the diffusion propagator (radial and angular part) is achieved by means of the so called Spherical Polar Fourier (SPF) basis functions. This basis is the combination of the angular functions $Y_{m}^{l}$ and the radial functions $R_{n}$ expressed as

$$
\Psi_{n \ell m}(r, \theta, \phi)=R_{n}(r) Y_{m}^{\ell}(\theta, \phi),
$$

\section{THE METHOD}

\subsection{Analytical EAP reconstruction}

In this section, we describe a method to accurately reconstruct the EAP from randomly undersampled measurements. The EAP can be estimated by a truncated linear combination of the SPF basis functions $\Psi_{n \ell m}$

$$
P(r, \theta, \phi)=\sum_{n=0}^{N} \sum_{\ell=0}^{L} \sum_{m=-\ell}^{\ell} c_{n \ell m} \Psi_{n \ell m}(r, \theta, \phi),
$$

where $c_{n \ell m}$ are the SPF transform coefficients. For a given SH order $L$ and a GL order $N$, the total number of SPF coefficients is $n_{c}=(1 / 2)(N+1)(L+1)(L+2)$.

Suppose $n_{q}$ is the number of measurement samples, $\mathbf{P} \in$ $\mathcal{R}^{n_{q}}$ a vector representing the EAP, $\mathbf{c} \in \mathcal{R}^{n_{c}}$ a vector of the SPF coefficients $c_{n \ell m}$ and $\Psi \in \mathcal{R}^{n_{q} \times n_{c}}$ the matrix constructed with the SPF basis functions

$$
\boldsymbol{\Psi}=\left(\begin{array}{ccc}
\Psi_{n \ell m}\left(r_{1}, \theta_{1}, \phi_{1}\right) & \cdots & \Psi_{N L L}\left(r_{1}, \theta_{1}, \phi_{1}\right) \\
\vdots & \ddots & \vdots \\
\Psi_{n \ell m}\left(r_{n_{q}}, \theta_{n_{q}}, \phi_{n_{q}}\right) & \cdots & \Psi_{N L L}\left(r_{n_{q}}, \theta_{n_{q}}, \phi_{n_{q}}\right)
\end{array}\right)
$$

We can write equation 10 as an overdetermined linear system

$$
\mathbf{P}=\Psi \mathbf{c}
$$

In order to find $\mathbf{c}$, we solve the convex optimization problem, specified in equation 4 using the SPF basis as the sparse transform, i.e. the coefficients are $c_{n \ell m}=\left\langle P, \Psi_{n \ell m}>\right.$. However, we don't need to explicitly compute these coefficients. Moreover, in Eq. 1, we see that the signal attenuation, after normalization $\mathbf{E}$, is the Fourier transform of the EAP 
$\mathbf{P}$, i.e. $\mathbf{E}=\mathcal{F}(\mathbf{P})$. Hence, in the SPF formalism, we have $\mathbf{E}=\mathcal{F}(\mathbf{\Psi} \mathbf{c})$. We call $\mathbf{E}_{u} \in \mathcal{R}^{n_{u}}$ the randomly acquired attenuation signal, $n_{u}$ being the number of undersampled samples. $\mathcal{F}_{u}$ is the undersampled Fourier transform where only the corresponding acquired coefficients remain after applying the Fourier transform. After rearranging equation 4, we obtain a new functional to minimize

$$
\operatorname{argmin}_{c} J(c)=\left\|\mathcal{F}_{u}(\Psi c)-E_{u}\right\|_{2}^{2}+\lambda\|c\|_{1} .
$$

Reference [13] reviews some results of CS-based reconstruction of the EAP when no sparse transform is applied. Note that modelling the propagator in SPF basis and then computing its Fourier transform is computationally expensive, especially in an iterative reconstruction scheme. Therefore, it would be of great interest to replace these two operations with a closed form solution.

In [12] the authors proved, after estimating the attenuation signal in the SPF family of functions, that it is possible to model the propagator in a dual Fourier basis using the same transform coefficients. In other words, this method can be resumed as follows : let $X$ be a signal in spherical coordinates and $a$ the coefficients of $X$ with respect to the SPF basis. Now, we consider the inverse Fourier transform $x=\mathcal{F}^{-1}(X)$. Reference [12] gives a SPF-dual (dSPF) basis in which $x$ can be modelled using the same coefficients $a$. It is an elegant closed form to compute an EAP when the corresponding diffusion signal is described by SPF coefficients. We cannot use directely this relation in our problem. However, we will see in the next paragraph how to take advantage of this relation.

Let us focus on Eq. 1, i.e. $\mathbf{E}(\mathbf{q})=\mathcal{F}(\mathbf{P}(\mathbf{R}))$. Because the attenuation signal is antipodally symmetric, i.e. $\mathrm{E}(\mathrm{q})=\mathrm{E}(-$ q), Eq. 1 is equivalent to

$$
E(\mathbf{q})=\int P(\mathbf{r}) \exp (2 \pi i \mathbf{q} \cdot \mathbf{R}) d \mathbf{R},
$$

i.e. $\mathbf{E}(\mathbf{q})=\mathcal{F}^{-1}(\mathbf{P}(\mathbf{R}))=\mathcal{F}(\mathbf{P}(\mathbf{r}))$, where $\mathbf{q}$ and $\mathbf{R}$ are both $3 \mathrm{D}$-vectors that respectively represent the effective gradient direction and the displacement direction.

Hence the same relation as in [12] holds while modelling the EAP with respect to the SPF basis. Now, suppose we have the SPF coefficients $\left\{c_{n \ell m}, n=0, \ldots, N, l=0, \ldots, L, m=\right.$ $-l, \ldots, l\}$ used to model $\mathbf{P}$, the corresponding attenuation signal can be reconstructed, in spherical coordinates, in the SPFd family of functions, $\left\{\Phi_{n \ell m}, n=0, \ldots, N, l=0, \ldots, L, m=\right.$ $-l, \ldots, l\}$ as

$$
\begin{aligned}
E(q, \theta, \varphi) & =\mathcal{F}^{-1}\left(\sum_{n=0}^{N} \sum_{\ell=0}^{L} \sum_{m=-\ell}^{\ell} c_{n \ell m} \Psi_{n \ell m}(r, \theta, \phi)\right) \\
& =\mathcal{F}\left(\sum_{n=0}^{N} \sum_{\ell=0}^{L} \sum_{m=-\ell}^{\ell} c_{n \ell m} \Psi_{n \ell m}(r, \theta, \phi)\right) \\
& =\sum_{n=0}^{N} \sum_{\ell=0}^{L} \sum_{m=-\ell}^{\ell} c_{n \ell m} \Phi_{n \ell m}(q, \theta, \phi)
\end{aligned}
$$

where $\mathcal{F}$ and $\mathcal{F}^{-1}$ are respectively the direct and inverse
Fourier transform, $q=|\mathbf{q}|$ is the norm of the effective gradient $\mathbf{q}$ in $\mathrm{q}$-space and $\theta, \varphi$ the direction angles. The SPFd family of function are expressed, for $n=0, \ldots, N, l=$ $0, \ldots, L, m=-l, \ldots, l$, as

$$
\Phi_{n \ell m}(q, \theta, \phi)=4(-1)^{\ell / 2} \frac{\zeta^{\ell / 2+3 / 2} \pi^{\ell+3 / 2} q^{\ell}}{\Gamma(l+3 / 2)} \gamma(\zeta, q) Y_{m}^{\ell}(\theta, \phi),
$$

with

$$
\begin{aligned}
\gamma(\zeta, q) & =\left[\frac{2^{\ell} n !}{\zeta^{\frac{3}{2}} \Gamma\left(n+\frac{3}{2}\right)}\right]^{\frac{1}{2}} \sum_{k=0}^{n} \frac{(-1)^{k}}{k !}\left(\begin{array}{c}
n+\frac{1}{2} \\
n-k
\end{array}\right) 2^{k} \ldots \\
& \cdots \Gamma\left(\frac{\ell}{2}+k+\frac{3}{2}\right) 1 F 1\left(\frac{2 k+l+3}{2}, l+\frac{3}{2},-2(\pi r)^{2} \zeta\right),
\end{aligned}
$$

We can write equation 15 as an over determined linear system

$$
\mathbf{E}=\mathcal{F}^{-1}(\Psi \mathbf{c})=\mathcal{F}(\Psi \mathbf{c})=\mathbf{\Phi} \mathbf{c}
$$

where $\mathbf{E} \in \mathcal{R}^{n_{q}}$ is a vector representing the attenuation signal, $\mathbf{c} \in \mathcal{R}^{n_{c}}$ the vector of the SPF coefficients $c_{n \ell m}$ and $\boldsymbol{\Phi} \in \mathcal{R}^{n_{q} \times n_{c}}$ matrix constructed with the SPFd basis functions in the same manner as described in (11).

Letting $\boldsymbol{\Phi}_{u} \in \mathcal{R}^{n_{u} \times n_{c}}$ be the randomly undersampled version of $\boldsymbol{\Phi}$ operator, we can rewrite the problem described in equation 13

$$
\operatorname{argmin}_{\mathbf{c}} J(\mathbf{c})=\left\|\boldsymbol{\Phi}_{\mathbf{u}} \mathbf{c}-\mathbf{E}_{\mathbf{u}}\right\|_{2}^{2}+\lambda\|\mathbf{c}\|_{1} .
$$

Remark that the problem no longer needs the computation of a Fourier transform. Instead, we remplaced it by a simple matrix multiplication. Eq. (19) searches for the EAP coefficients with respect to the SPF basis, i.e. we can compute a continuous version of the true propagator. Using the same coefficients, we can as well model the attenuation signal with respect to the SPFd basis functions. The next section shows an analytical solution to reconstruct the ODF in terms of spherical harmonics.

\subsection{ODF feature extraction}

The orientation distribution function is a function on the unit sphere describing the probability averaged over the voxel that a particle will diffuse into a given solid angle. The diffusion ODF contains the full angular information of the EAP and is defined as [7, 22],

$$
\Psi(\theta, \phi)=\int_{0}^{\infty} P(r, \theta, \phi) r^{2} d r,
$$

where $(\theta, \phi)$ obey physics convention $(\theta \in[0, \pi], \phi \in$ $[0,2 \pi])$.

Using $\int_{0}^{\infty} \exp (-s x) x^{\alpha} L_{n}^{\alpha}(x) d x=\frac{\Gamma(\alpha+n+1)(s-1)^{n}}{n ! s^{\alpha+n+1}}$ [12], we prove that one can rewrite equation 20 in the SPF formalism as, 


$$
\Psi(\theta, \phi)=\sum_{l=0}^{L} \sum_{m=-l}^{l}\left(\sum_{n=0}^{N} 2(-1)^{n} c_{n l m}\left[\frac{\Gamma(n+3 / 2)}{n !}\right]\right) Y_{l}^{m}(\theta, \phi) .
$$

This is an important formula allowing to analytically estimate the ODF in terms of spherical harmonic functions. Due to the lack of space, the derivation is omitted here.

\section{EXPERIMENTATION}

For each experiment, we construct the SPF dual basis with $N=4$ and $L=8$ so as to obtain a sparse basis (the number of coefficients is $n_{c}=225$ ). Knowing the sampling coordinates, the basis matrix can be computed offline. We solve the problem by means of a Fast Iterative Shrinkage-Thresholding Algorithm (FISTA) [23], an iterative algorithm where each iteration involves a shrinkage step. We chose this algorithm because of its speed. In a Matlab implementation, the technique proposed takes a few seconds (usually less than 0.25 seconds) to reconstruct the vector of coefficients for one voxel, on a Intel Core 2 Duo CPU at $2.8 \mathrm{GHz}$. Compare to [13], this technique significantly shorten the reconstruction time by about 80 times. This is important when dealing with thousands of voxel (And it is ussually the case). We evaluate $\zeta$ in such a way that the first order function of GL basis fits a typical Gaussian based signal $P(r)=\exp \frac{-r^{2}}{4 \tau D}$. For $4 \pi^{2} \tau=1$ and $D=0.7 e-3 \mathrm{~mm}^{2} / \mathrm{s}$, we set $\zeta=\frac{D}{2 \pi^{2}}=3.5462 e-5$ in all experiments.

\subsection{Synthetic data}

We review the different potentialities of the proposed analytical solution on the reconstruction of synthetic data features generated using a multi-tensor model. The normalized diffusion signal at a shell with $b=4 \pi^{2} \tau q^{2}$ is thus described, by $F$ fibres, as $E(\mathbf{u})=\sum_{f=1}^{F} p_{f} \exp \left(-4 \pi^{2} \tau q^{2} \mathbf{u}^{T} D_{f} \mathbf{u}\right)$ where a fibre $f$ is defined by a tensor matrix $D_{f}$ and weight $p_{f}$. $q$ denotes the norm of the effective gradient and $\mathbf{u}$ is a unitary vector in Cartesian coordinate. The analytical ground truth of the EAP for any radius $\mathrm{R}$ is then given by $P(R \mathbf{r})=$ $\sum_{f=1}^{F} p_{f} \frac{1}{\sqrt{(4 \pi \tau)^{3}\left|D_{f}\right|}} \exp \left(\frac{-R^{2} \mathbf{r}^{T} D_{f}^{-1} \mathbf{r}}{4 \tau}\right)$ with $\mathbf{r}$ a unitary vector in Cartesian coordinate. We can also check the ODF feature using solid angle closed form expression [7] , $\psi(r)=$ $\sum_{f=1}^{F} p_{f} \frac{1}{4 \pi\left|D_{f}\right|^{\frac{1}{2}}\left(\mathbf{r}^{T} D_{f}^{-1} \mathbf{r}\right)^{\frac{3}{2}}}$

We reconstruct the ODF and the EAP profiles at radius $R=12,15,18 \mu \mathrm{m}$ for three fibre configurations: one fibre, $90^{\circ}$ and $60^{\circ}$ - crossing fibres. We choose a tensor matrix whose eigenvalues are $[0.3,1.7,0.3] \mathrm{e}-3 \mathrm{~mm}^{2} / \mathrm{s}$ and rotate it according to the fibre configuration. For each configuration, we add Rician noise with $\mathrm{SNR}=25,20$ and 15 . The aim is to show that it is still possible to reconstruct EAP features with very few samples. Therefore, we randomly take 80 measurements

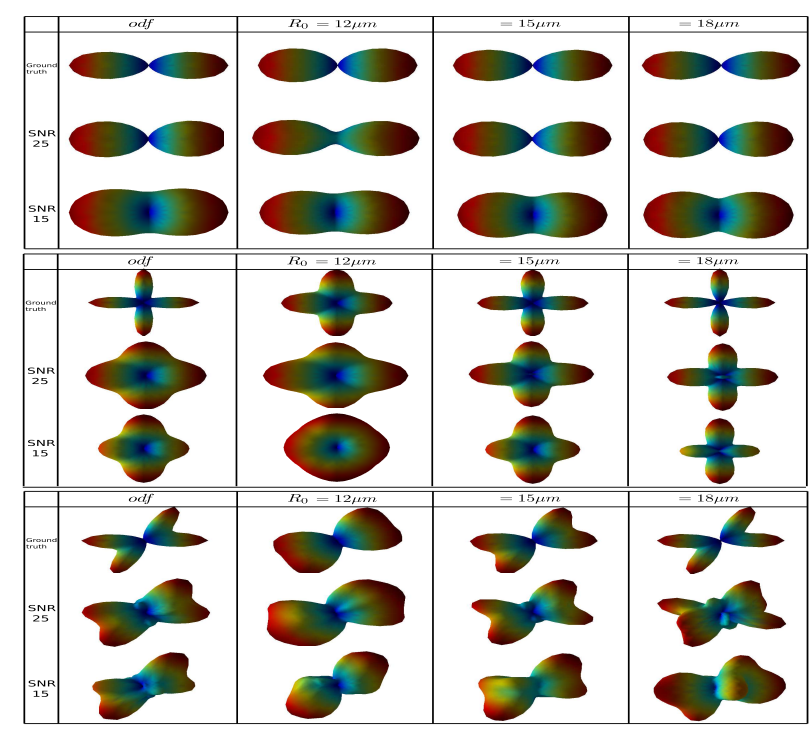

Fig. 1. Synthetic data results with one fibre, $90^{\circ}$ and $60^{\circ}$ crossing fibres configurations respectively from the top to the bottom. We show from the left to the right : the estimated ODF and the EAP profiles at radius $R=12,15,18 \mu \mathrm{m}$.

\begin{tabular}{|c|c|c|c|}
\hline \multicolumn{2}{|c|}{} & CS method & $L_{2}$ regularization \\
\hline SNR=25 & one fibre & $\left(100.0 \%, 0.17^{\circ}\right)$ & $\left(100.0 \%, 0.34^{\circ}\right)$ \\
& $90^{\circ}$ - crossing fibres & $\left(95.3 \%, 2.46^{\circ}\right)$ & $\left(88.0 \%, 4.67^{\circ}\right)$ \\
& $60^{\circ}$ - crossing fibres & $\left(85.3 \%, 5.11^{\circ}\right)$ & $\left(84.7 \%, 6.47^{\circ}\right)$ \\
\hline SNR=20 & one fibre & $\left(100.0 \%, 0.75^{\circ}\right)$ & $\left(100.0 \%, 0.84^{\circ}\right)$ \\
& $90^{\circ}$ - crossing fibres & $\left(90.0 \%, 3.20^{\circ}\right)$ & $\left(88.0 \%, 5.32^{\circ}\right)$ \\
& $60^{\circ}$ - crossing fibres & $\left(74.7 \%, 6.58^{\circ}\right)$ & $\left(75.0 \%, 6.59^{\circ}\right)$ \\
\hline SNR=15 & one fibre & $\left(91.2 \%, 3.06^{\circ}\right)$ & $\left(100.0 \%, 2.79^{\circ}\right)$ \\
& $90^{\circ}$ - crossing fibres & $\left(81.1 \%, 6.10^{\circ}\right)$ & $\left(86.0 \%, 6.50^{\circ}\right)$ \\
& $60^{\circ}$ - crossing fibres & $\left(56.3 \%, 10.73^{\circ}\right)$ & $\left(62.7 \%, 9.47^{\circ}\right)$ \\
\hline
\end{tabular}

Table 1. Percentage of correct number of detected maximum and the mean of angular error on the estimated ODF

spread on 3 shells with b values $1000,2000,3000 \mathrm{~s} / \mathrm{mm}^{2}$. Random means several sampling schemes are possible so, we selected it as follow : On 100 sampling schemes generated, we kept the one that leads to the best results. We set $\lambda=0.01$ when $\mathrm{SNR}=25$ and 20 , and $\lambda=0.015$ when $\mathrm{SNR}=15$.

Some experiments with SNR=25 and 15 (fig. 1 ) showed that our CS-based method works well in recovering angular information. Indeed, it is easy to detect maxima on the ODF.We also see that EAP profiles are well approximated for one fibre and two $90^{\circ}$ - crossing fibres, especially with $\mathrm{SNR}=25$. With the $60^{\circ}$ - crossing fibres configuration, the reconstruction still gives good results considering the amount of noise and the fact we take only 80 samples.

A more thorough and quantitative evaluation is perform on the ODF feature reconstruction. Table 1 presents the percentage of correct number of detected maximum and the mean of angular error of the estimated ODF on 1000 trials, with our method and a least square method with $l_{2}$ regular- 
ization. In the latter technique, we regularly take 80 samples and the same regularization parameters for the radial $\lambda_{N}$ and angular $\lambda_{L}$ parts, i.e $\lambda_{N}=\lambda_{L}=1 e-8$ when $\mathrm{SNR}=25$ and 20, and $\lambda_{N}=\lambda_{L}=6 e-8$ when $\mathrm{SNR}=15$. These results shows that our method is better for SNR=25 and 20 but doesn't outperform $l_{2}$ regularization at low SNR. This is due to the different effects that provide $l_{1}$ and $l_{2}$ regularizations. $l_{1}$ promotes sparsity whereas $l_{2}$ is known as a smoothing (so denoising) regularization. In brief, our method accept a decrease of the number of samples with better results than the classical $l_{2}$-norm method as long as the SNR remains higher than 15 .

\subsection{Phantom data}

We prove the effectiveness of our method in reconstructing the propagator on a phantom data. This phantom was used in the fibres cup contest, at the MICCAI conference in London in 2009, to benchmark tractography algorithms [24]. These diffusion measurements, devoted to evaluate HARDI models, are known to be especially difficult in recovering directional information because of their low anisotropies. We have 192 attenuation signal samples equally spread on 3 shells with bvalues of 650,1500 , and $2000 \mathrm{~s} / \mathrm{mm}^{2}$. A first reconstruction is achieved after keeping all the coefficients, i.e. 192, and a second one after randomly considering only 80 samples along an uniform distribution in the entire data set. Note that, in this way, each shell contains approximately the same number of measurements, thus lower is the b-value and higher is the sample density on the corresponding shell. Reference [13] shows that this kind of behaviour improves numerical reconstruction. For every experiment the regularization parameter is set to $\lambda=0.02$. Figure 2 shows the experimental results for the ODF estimation and the EAP values at a radius $R=15 \mu \mathrm{m}$. For each case, we focus our attention on a crossing region ( $A$ and $B$ on the figure). Our CS-based reconstruction works well on this data even with 80 samples. Our goal to highly reduce the number of measurements, while keeping the important information about the diffusion, is achieved. Note that the reconstruction works for a higher range of radius, however, due to the lack of space, we cannot show these results.

\section{CONCLUSION}

In this paper, we have described a new and efficient compressedsensing based method to accurately reconstruct the EAP in term of Spherical Polar Fourier functions from few diffusion DW-MRI images. We provided an efficient solution to estimate the EAP at any radius $R$ and proposed also an analytical solution to reconstruct the ODF function. We proved the effectiveness of our method in reconstructing the EAP and the ODF on both noisy multiple q-shell synthetic data and phantom data. However, the results are sensitives to the sampling scheme used for the acquisition so we are working on efficient way to sample the diffusion.

Acknowledgements: This work was partially supported by the ANR project NucleiPark and the France-Parkinson association.

\section{REFERENCES}

[1] Denis Le Bihan and E. Breton, "Imagerie de diffusion it in vivo par résonnance magnétique nucléaire," $C R$ Académie des Sciences, , no. 301, pp. 1109-1112, 1985.

[2] K.D. Merboldt, W. Hanicke, and J. Frahm, "Selfdiffusion nmr imaging using stimulated echoes," $J$. Magn. Reson., vol. 64, pp. 479-486, 1985.

[3] D.G. Taylor and M.C. Bushell, "The spatial mapping of translational diffusion coefficients by the nmr imaging technique," Phys. Med. Biol., vol. 30, pp. 345-349, 1985.

[4] E.O. Stejskal and J.E. Tanner, "Spin diffusion measurements: spin echoes in the presence of a time-dependent field gradient," Journal of Chemical Physics, vol. 42, pp. 288-292, 1965.

[5] D. Tuch, “Q-ball imaging," Magnetic Resonance in Medicine, vol. 52, no. 6, pp. 1358-1372, 2004.

[6] Maxime Descoteaux, Elaine Angelino, Shaun Fitzgibbons, and Rachid Deriche, "Regularized, fast, and robust analytical q-ball imaging," Magnetic Resonance in Medicine, vol. 58, no. 3, pp. 497-510, 2007.

[7] Iman Aganj, Christophe Lenglet, and Guillermo Sapiro, "Odf reconstruction in q-ball imaging with solid angle consideration,", in Proceedings of the Sixth IEEE International Symposium Biomedical Imaging, Boston, MA,, June 2009.

[8] O. Michailovich and Y. Rathi, "On approximation of orientation distributions by means of spherical ridgelets," Image Processing, IEEE Transactions on, vol. 19, no. 2, pp. 461-477, 2010.

[9] Evren Özarslan, T.M. Shepherd, Baba C. Vemuri, S.J. Blackband, and Thomas H. Mareci, "Resolution of complex tissue microarchitecture using the diffusion orientation transform (dot)," NeuroImage, vol. 31, no. 3, pp. 1086-1103, 2006.

[10] Maxime Descoteaux, Rachid Deriche, Denis Le Bihan, Jean-François Mangin, and Cyril Poupon, "Multiple q-shell diffusion propagator imaging," Medical Image Analysis, July 2010.

[11] H.E. Assemlal, D. Tschumperlé, and L. Brun, "Efficient computation of pdf-based characteristics from diffusion mr signal," Medical Image Computing and ComputerAssisted Intervention, , no. 70-78, 2008. 


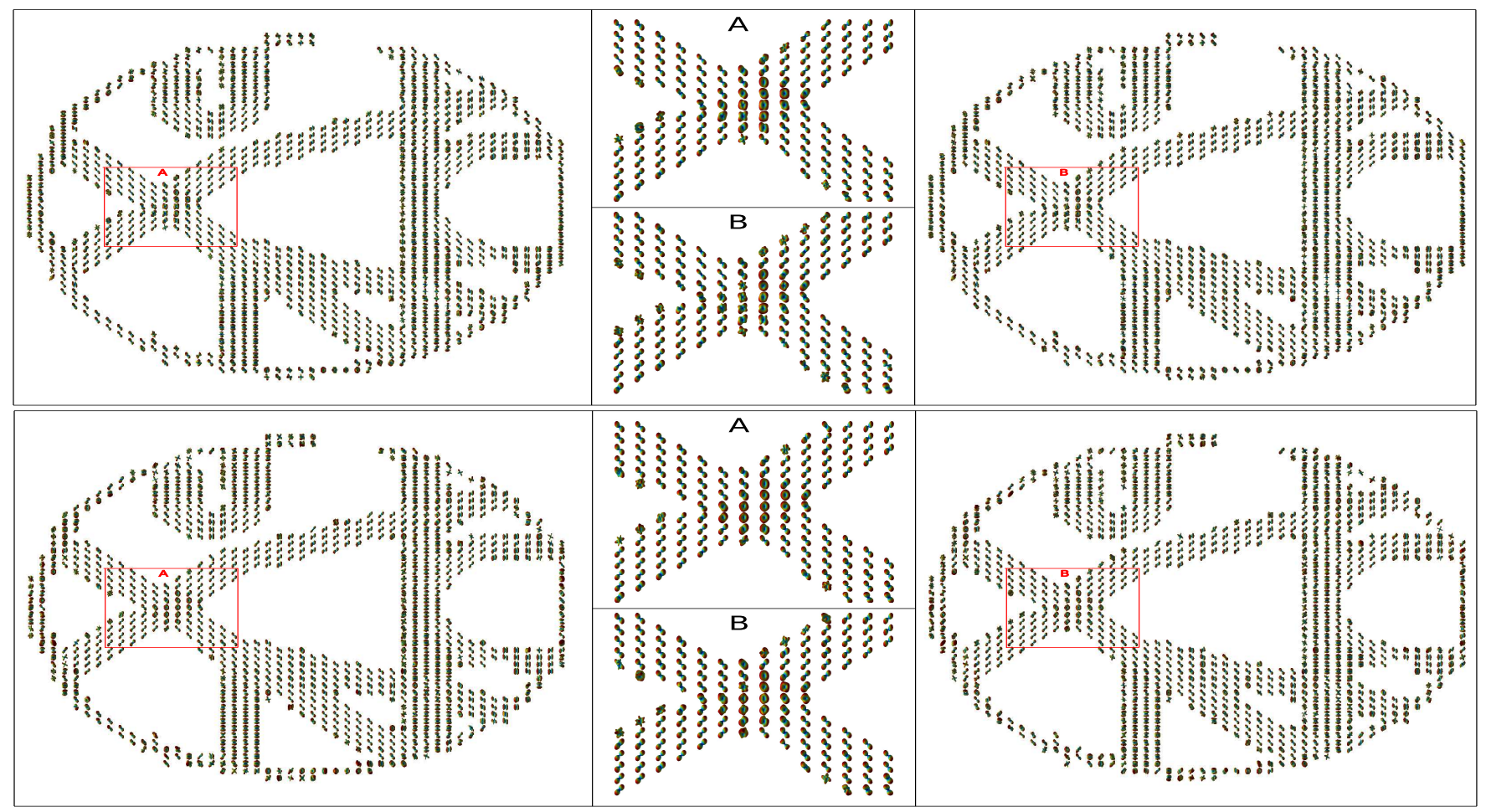

Fig. 2. Phantom data. Top : ODF estimation with 192 samples (left) and 80 samples (right). Bottom : reconstruction of $P(R \mathbf{r})$ at radius $R=15 \mu \mathrm{m}$ with 192 samples (left) and 80 samples (right). Frame $A$ and $B$ are both zoom on a crossing fibres region.

[12] Jian Cheng, Aurobrata Ghosh, Tianzi Jiang, and Rachid Deriche, "Model-free and analytical eap reconstruction via spherical polar fourier diffusion mri," in Medical Image Computing and Computer-Assisted Intervention - MICCAI, 2010, vol. 6361, pp. 590-597.

[13] Sylvain Merlet and Rachid Deriche, "Compressed sensing for accelerated eap recovery in diffusion mri," in Proceedings Computational Diffusion MRI - MICCAI Workshop, Sept. 2010.

[14] O. Michailovich, Y. Rathi, and S. Dolui, "Spatially regularized compressed sensing of diffusion mri data," Arxiv preprint arXiv:1009.1889, 2010.

[15] M. Lustig, D. Donoho, and J.M. Pauly, "Sparse mri: The application of compressed sensing for rapid $\mathrm{mr}$ imaging," Magnetic Resonance in Medicine, vol. 58, no. 6, pp. 1182-1195, 2007.

[16] W. Guo and W Yin, "Edgecs: Edge guided compressive sensing reconstruction," Rice CAAM Report TR10, vol. 2, pp. 30, 2010.

[17] A. Ganesh, VR Edward, et al., "Reordering for improved constrained reconstruction from undersampled k-space data," International Journal of Biomedical Imaging, 2008.
[18] R. Chartrand, "Fast algorithms for nonconvex compressive sensing: Mri reconstruction from very few data," IEEE International Symposium on Biomedical Imaging (ISBI), 2009.

[19] David L. Donoho, "Compressed sensing," IEEE Trans on Information Theory, vol. 52, no. 4, pp. 1, 2004.

[20] D.L. Donoho, "Method and apparatus for compressed sensing," Jan. 2010, US Patent 7,646,924.

[21] S. Erturk and TJ Dennis, "3d model representation using spherical harmonics," Electronics Letters, vol. 33, no. 11, pp. 951-952, 2002.

[22] A. Tristan-Vega, SC.F. Westin, and S. Aja-Fernandez, "Estimation of fiber orientation probability density functions in high angular resolution diffusion imaging," NeuroImage, vol. 47, no. 2, pp. 638-650, Aug. 2009.

[23] M. Zibulevsky and M. Elad, "L1-12 optimization in signal and image processing," IEEE Signal Processing Magazine, vol. 27, no. 3, May 2010.

[24] C. Poupon, B. Rieul, I. Kezele, M. Perrin, F. Poupon, and J-F. Mangin, "New diffusion phantoms dedicated to the study and validation of hardi models," Magnetic Resonance in Medicine, vol. 60, pp. 1276-1283, 2008. 